\title{
The Fundamental Principles of Official Statistics in the Statistics Centre Abu Dhabi (SCAD)
}

\author{
Dr. Mohammed Al Rifai \\ Statistics Centre - Abu Dhabi, P.O. Box: 6036 Abu Dhabi, UAE
}

\begin{abstract}
The Fundamental Principles of Official Statistics were adopted by the United Nations Statistical Commission in 1994 and are now universally agreed as a comprehensive framework for the mission of National Statistical Offices. It gives a set of fundamental values and principles, which NSOs and other data producers should have in order for the public to have trust in the official statistics they produce.Statistics Centre - Abu Dhabi (SCAD) is the main authorized body concerned with official statistics in the Emirate of Abu Dhabi. It is responsible for the collection, classification, storage, analysis and dissemination of official statistics covering social, demographic, economic, environmental and cultural indicators. It has considered the fundamental principles of official statistics as a foundation stone in producing the Emirate's official statistics, to satisfy the highest level of data quality standards and to gain the trust of the public with its statistical production. This article will discuss the institutional, organizational, and technical activities that have been undertaken by SCAD to comply with the fundamental principles of official statistics. It will highlight the principles and implementations adopted by SCAD with examples.
\end{abstract}

Keywords: Fundamental principles, official statistics, Quality.

DOI: $10.7176 / \mathrm{JESD} / 10-20-09$

Publication date:October $31^{\text {st }} 2019$

\section{Introduction}

1.1 Abu Dhabi

Abu Dhabi is the federal capital of the United Arab Emirates (UAE) and the largest of the seven emirates. Geographically, Abu Dhabi lies on the borders with the Kingdom of Saudi Arabia, the Sultanate of Oman, and the Arabian Gulf. Over the past 40 years, Abu Dhabi has experienced significant population growth and economic development.

To manage the growth and prosperity of the Emirate, the Government of Abu Dhabi required an official agency that could provide statistics for decision-making and policy setting.

\subsection{Statistics Centre - Abu Dhabi}

Statistics Centre - Abu Dhabi (SCAD) was established in accordance with Law \#7 for the year 2008. SCAD is responsible for the collection, classification, storage, analysis and dissemination of official statistics covering social, demographic, economic, environmental and cultural indicators.

As a young statistical office, SCAD is in the fortunate position of being able to implement best practices from international bodies and leading National Statistical Organizations (NSOs). SCAD is aiming to be a world leader in innovative and efficient methods for data collection, analysis and dissemination.

\subsection{Background to the fundamental Principals of Official Statistics}

The United Nations developed a set of fundamental principles for official statistics, in order for the statistical systems in the countries to have the confidence of national and international users of statistics. In other words, the fundamental principles are a set of governing values developed by the United Nations to ensure robust and independent statistics from the world's national statistics offices.

According to their relevance, these principles should be treated as a key step in creating a solid policy platform for the governing of National Statistics Offices. The principles are not designed to replace legislation, but to operate alongside comprehensive statistics legislation as a powerful tool to ensure the robust operation of the national statistics systems, and to assure users of the transparency of official information. Further, the principles will provide guidance to, and inform discussions between, the national statistical systems, government, data users, and respondents; who are the main players in the official statistics production.

After the principles adopted by the Conference of European Statisticians and the Economic Commission for Europe, statisticians in other parts of the world began to realize that these principles had a wider significance. In that context, an international discussion process was started, ending with the adoption of the Fundamental Principles by the United Nations Statistical Commission, the highest statistical authority in the world. The 10 principles are as follows:

Principle 1 - Relevance, independence and equal access: Official statistics provide an indispensable element in the information system of a democratic society, serving the Government, the economy and the public with data 
about the economic, demographic, social and environmental situation. To this end, official statistics that meet the test of practical utility are to be compiled and made available on an impartial basis by official statistical agencies to honor citizens' entitlement to public information.

Principle 2 - Professional standards and ethics: To retain trust in official statistics, the statistical agencies need to decide according to strictly professional considerations, including scientific principles and professional ethics, on the methods and procedures for the collection, processing, storage and presentation of statistical data.

Principle 3 - Accountability and transparency: To facilitate a correct interpretation of the data, the statistical agencies are to present information according to scientific standards on the sources, methods and procedures of the statistics.

Principle 4 - Prevention of misuse: The statistical agencies are entitled to comment on erroneous interpretation and misuse of statistics.

Principle 5 - Cost-effectiveness: Data for statistical purposes may be drawn from all types of sources, be they statistical surveys or administrative records. Statistical agencies are to choose the source with regard to quality, timeliness, costs and the burden on respondents.

Principle 6 - Confidentiality: Individual data collected by statistical agencies for statistical compilation, whether they refer to natural or legal persons, are to be strictly confidential and used exclusively for statistical purposes.

Principle 7 - Legislation: The laws, regulations and measures under which the statistical systems operate are to be made public.

Principle 8 - National co-ordination: Coordination among statistical agencies within countries is essential to achieve Consistency and efficiency in the statistical system.

Principle 9 - Use of international Standards: The use by statistical agencies in each country of international concepts, Classifications and methods promotes the consistency and efficiency of statistical systems at all official levels.

Principle 10 - International Statistical co-operation: Bilateral and multilateral cooperation in statistics contributes to the improvement of systems of official statistics in all countries.

\section{The Principles implementation in Statistics Centre - Abu Dhabi}

A set of legislations, policies, actions and procedures were undertaken to serve the implementation of the fundamental principles of official statistics in SCAD. In addition to, different working frames and technical documents were developed, such as the assessment quality frame, a confidentiality policy, SCAD's code of practice, SCAD's code of ethics, etc.

\section{Principle 1 - Relevance, independence and equal access}

The relevance of official statistics produced by SCAD is built through coordination with, and requests from, data users, to help compile the data; which serve the Government and the public informing the economic, demographic, social and environmental situation. To achieve this:

- National technical committee has been formed by SCAD, including client representative from government entities. This committee holds a number of technical meetings throughout the year to discuss statistical issues and requests from clients.

- $\quad$ Every year, SCAD invites all clients in the Abu Dhabi government to an annual forum to discuss the plans and projects related to statistical production, in addition to discussing their issues and requests.

- As a first step in the production process, i.e. prior to the data collection and production of the relevant statistics, SCAD discusses all the related processes, variables and methodologies with statistic requesters.

With respect to impartiality, SCAD compiles its official statistics based on clear methodologies built on international practices and standards. The implemented methodologies are available to the public in the methodology section of the SCAD website and are attached to its statistical publications. All data user groups have the right to see all the details.

Further, the statistics produced by SCAD are published impartially on a frequent basis, and most decisions related to what and when most of the information is released are free from political interference and government manipulation; which give users more confidence in the authenticity of official information.

The publication of the most of periodical statistics in SCAD like, Prices, foreign trade, divorce statistics, etc. is independent of administrative resolutions from the management or other concerned entities in the government. Most of statistics produced in SCAD are released electronically through the website; therefore, all groups of data users have the right to equal access to the produced statistics.

\section{Principle 2 - Professional Standards and Ethics}

In order to attain professionalism in data production at SCAD, a set of procedures was undertaken by SCAD, technically, all statistical methods, procedures of data collection, processing, and presentation of statistical data were designed in professional way according to the best statistical methods and recommendations.

Furthermore, SCAD prepared a Standard Operating Procedures Manual on Core Statistical Production Processes, It details the key operational policies and guidelines applicable for the execution and decision making 
within each statistical process. This manual is a general process documentation and hence an element in the implementation of GSPBM. Its main objective is to provide guidance to the employees of SCAD on how to carry out processes within the data and statistics management process platform in the SCAD process framework and to improve transparency communication, accountability, efficiency and controls.

With respect to ethics, SCAD issued a code of ethics charter, which sets out the occupational ethics governing statistical practice and aims to establish sound ethical principles to regulate and monitor statistical work in the Emirate. Such principles follow best statistical standards and practices within an ideal work environment that stimulates perfection and excellence.

The main objective of the charter is to promote the moral values of staff working in the field of statistics and document the ethical principles of the statistical work in Abu Dhabi, In addition to Develop the best statistical practices and strengthen public confidence in the statistical system of the Emirate.

\section{Principle 3 - Accountability and Transparency}

For correct interpretation and better understanding of published statistical data, the methodology department has constructed a methodology web page linked to SCAD website. It consists of three main sections. The methods and standards section contains information on the statistical methods and standards used for SCAD surveys and administrative sources. The statistical classifications section provides updated information on the classifications used, to give a common language to interpret, arrange, and compare statistics at national and international levels. The data quality section describes the statistical quality management and quality assurance framework within SCAD, according to the "SCAD Data Quality Assessment Framework". It explains how SCAD manages the quality of its data and products to provide independent, objective and trustworthy statistics.

In addition to the methodology webpage, SCAD prepared the "Abu Dhabi Statistical Manual" which describes both the organizational statistical work in the Abu Dhabi statistical system and all of the statistical production in the statistical system.

Within its remit of managing the technical statistical work in the Abu Dhabi statistical system, SCAD prepared a set of technical guides to unify, interpret and further understanding of statistics in the Emirate. The most important guides were "Statistical Survey Implementation Guide", "Statistical Indicators Guide", "Statistical Data Editing Guide". "Quality Guide of Survey data", and "Quality Guide of administrative data.

The statistical metadata system was developed by SCAD to describe data and metadata, using professional and understandable methods, to the users. The system is based on policies and procedures for collecting statistical metadata for four statistical resources used in SCAD: datasets, variables, indicators, and quality statements.

\section{Principle 4 - Prevention of misuse}

This principal gives SCAD the mandate to ensure correct use of statistical information. The initiated "dissemination policy", gives SCAD reserves the right to comment on erroneous interpretation, or misuse of statistical information, for example when there is a serious error of fact or when the comment impinges on the reputation/credibility of SCAD and its statistics.

\section{Principle 5 - Cost-effectiveness}

According to law No (7) of the year 2008, the article (17) indicates that statistical works and activities by the Centre and activities in connection with the preparation and dissemination of official statistics shall be based on a set of primary principals, one of these is cost effectiveness.

In order to implement this principal, a set of actions has been undertaken by SCAD. We all know that the cost of the statistics mainly depend on the source of the data, whether sample surveys and census or administrative records. In the case of sample surveys there are two main factors to be taken into account, the costs and accuracy. During the last few years SCAD has used to carry out a series of analyses of the surveys aimed at reducing the samples size in order to reduce costs, whilst keeping the data accuracy within the acceptable ranges.

In addition, coordination and service level agreements were conducted with the data providers, mainly in business surveys, to provide SCAD data through email transfer. In order to facilitate data transfer, the questionnaires were revised and improved, in order to integrate accounting and statistical terminologies to enables the data providers to complete the form.

In order to improve the use of administrative data for statistical work in the Emirate of Abu Dhabi, a committee was formed to develop the strategy to work with the administrative records in order to unify and coordinate initiatives and efforts in the of development of administrative records.. Seven statistical registers have been targeted for development: population, buildings and housing units, business register, export and import register, agriculture register, national employed register, national un-employed register.

\section{Principle 6 - Confidentiality}

$\mathrm{SCAD}$ is accountable to the Abu Dhabi government to provide reliable information for sound decision making and understanding the economic and social status of the Emirate. SCAD is also accountable to the Abu Dhabi public and businesses to protect their identities. There is a duty of care to both the government and public - and it is within this space that confidentiality exist to protect respondents' identities and to provide data that is statistically meaningful and reliable for the government. 
Law $\mathrm{No}(7)$ of the year 2008, Article(4) requires that SCAD aims to provide accurate and reliable statistics in the social, economic, environmental, and other situations to decision and policy makers, the public, mass media, the business community, researchers and the international community, without prejudice to the confidentiality of individuals in the data. SCAD's work occurs in accordance with the provisions of this law and the data dissemination policy approved by the management council.

To achieve the aim in Article(4), Article(5) requires that SCAD shall observe that official statistics are collected prepared, and published using applicable procedures and instructions taking into account the privacy of the individuals and the confidentiality of individual data.

In addition, Article (17) considers statistical confidentiality as one of the primary principles in of its statistical works and activities.

From the technical perspective, SCAD has established "SCAD confidentiality policy", to provide with the legal, ethical and technical direction. As a result of reading this policy and implementing the guidelines within it, SCAD staff will understand their obligation to respondents and gain confidence in their understanding of the law and principles that dictate the necessity to have such rules in the first place.

\section{Principle 7 - Legislation}

SCAD has a comprehensive law No(7) of the year 2008 and related regulations governing the statistical work in the Emirate, In addition to organizational and technical policies related to the statistical operation, which organize the roles, responsibilities, duties and rights among the all parties, government, data users, data providers, public, etc. All of these regulations and policies, in addition to law No (7) are available at SCAD website and publications to users, and all the public. This is to ensure statistical providers, users, and respondents can be fully away of their rights, and their responsibilities.

\section{Principle 8 - National Co-ordination}

The Statistical System of Abu Dhabi consists of SCAD and other Government agencies within the Emirate that perform statistical activities on a regular basis.

Abu Dhabi law No(7) of the year 2008 requires all Government entities to provide SCAD with statistical information, to consult SCAD before starting any statistical project, to abide by the definitions, classifications and technical statistical standards set by SCAD and to obtain the Centre's consent before publishing results. In addition, the Law prescribes close cooperation between SCAD and the statistical agency at the Federal UAE level. Hence, SCAD should undertake surveys according to the Federal annual work program and on the basis of the methodologies, standards, classifications and definitions adopted by the Federal.

An effective and result-oriented relationship with the data providers and other key stakeholders is a critical success factor for SCAD's statistical work. The basic tool of a partnership arrangement with providers of administrative data or other partners in statistics production consists of Memoranda of Understanding (MOU) and Service Level Agreements (SLA) that set out the mechanisms and responsibilities for data transmission. SCAD had signed more than 50 Service Level Agreements with key stakeholders, including Abu Dhabi government, academic institutions, federal government, and statistics centers. In addition, SCAD holds an annual stakeholders' forum to discuss issues and understand their priorities. The outcome helps inform and develop an action plan to meet users' statistical requirements.

Based on the established "Charter of Cooperation, Service and Transparency", SCAD undertakes to jointly coordinate and constructively cooperate with all partners, stakeholders, departments, institutions and individuals, to develop the statistical system and to achieve the supreme interest of the Emirate of Abu Dhabi.

SCAD, along with its strategic partners in the Statistical System of Abu Dhabi (SSAD), plays a pivotal role in supporting the development process, decision-makers, and policy-makers in Abu Dhabi. As an important component of the SSAD, SCAD has developed "SCAD Code of Practice", to be implemented by all strategic partners.

Principle 9 - Use of International Standards

Comparability is an important dimension of quality. If data is not comparable, it loses a lot of its utility. If data loses its utility, the agency that produces it loses relevance.

Within the statistical framework and in accord with Law No (7) of the year 2008, SCAD has adopted the related international standards, manuals, and classifications to be used in the production of all of its statistical data. In addition, as a key coordinator of statistical work in the Emirate, SCAD produces guides and conducts ongoing training courses to the data producers in the Abu Dhabi Government in order to provide details on the standards to use and how to implement them. The methodology webpage at SCAD's website provides links to relevant international manuals and classifications that are used by SCAD.

Principle 10 - International Statistical Co-operation

The aim of this principal is to learn from and share best statistical practice. This aim contributes to the development and improvement of international classifications, methods, definitions and guidelines. SCAD Law (7) Article (5) indicates that SCAD should participate in statistics related to international cooperation, and share experience and expertise with the Arab and international scientific agencies and institutions working in the field of statistics. 
SCAD contributes to many statistical conferences and events around the world. In addition, the contribution of the Abu Dhabi Emirate in hosting the IAOS2016 emphasizes SCAD's work to support cooperative relations with international institutes and countries.

SCAD has established effective international co-operation with different regional and international statistical institutions around the world, including the GCC-STAT, UNSD, and Eurostat. In addition, SCAD regularly works with different national statistical offices around the world. Memoranda of understanding and agreements of cooperation have been signed with various national statistical offices in countries such Germany, Italy, Korea, Australia, etc.

These agreements have covered training courses in coordination with SCAD's statistical training institute STI, in addition to capacity and experiences building through exchange visits..

\section{Conclusions}

- The articles of the statistics law (7), from 2008, have the capacity to cover all the institutional and legislative aspects described in the fundamental principles of official statistics.

- $\quad$ SCAD has undertaken preparation of most of the documents, manuals, charters, and guides contributing to achieving the fundamental principles of official statistics in Abu Dhabi.

- Despite the achievement of most of the legislative and technical requirements included in the fundamental principles of official statistics, some aspects still need support to promote optimal achievement of the fundamental principles of official statistics.

\section{References}

Statistics Law No. 7, statistics Centre- Abu Dhabi 2008.

Fundamental Principles of Official Statistics (A/RES/68/261 from 29 January 2014), [online] available: http://unstats.un.org/unsd/dnss/gp/fundprinciples.aspx

Standard Operating Procedures Manual on Core Statistical Production Processes, Statistics Center Abu Dhabi 2011.

Charter of Professional Ethics, Statistics Center Abu Dhabi 2015.

Data Quality Assessment framework, Statistics Center Abu Dhabi 2011.

Survey Quality Manual, Statistics Center Abu Dhabi, 2016

QA Admin Data Manual Statistics Center Abu Dhabi, 2015

Dissemination policy, Statistics Center Abu Dhabi, Version 1.5, 2014.

Confidentiality Policy, Statistics Center Abu Dhabi, 2012.

The Charter of Cooperation, Service and Transparency, Statistics Center Abu Dhabi, 2013.

Code of Practice, Statistics Center Abu Dhabi, 2015. 